\title{
Characterization and modelling of EMI susceptibility in integrated circuits at high frequency
}

\author{
Ignacio Gil* and Raúl Fernández-García \\ Department of Electronic Engineering \\ UPC. Barcelona Tech \\ Colom 1, 08222 Terrassa, Spain \\ *e-mail: ignasi.gil@upc.edu
}

\begin{abstract}
In this paper an alternative method for characterizing and modelling the EMI susceptibility in integrated circuits at frequencies above $1 \mathrm{GHz}$ is presented. The PCB layout design is focused on the optimization of the impedance mismatch losses on the radio frequency interference injection path. The PCB has been tested with several commercial operational amplifiers and the methodology is validated through both electrical transmission line simulations and electromagnetic cosimulations.
\end{abstract}

Keywords-Immunity to Electromagnetic Interferences; Integrated Circuits; Operational Amplifier

\section{INTRODUCTION}

Over recent years, due to the increasing demand for multifunctional, multi-band wireless operation electronic devices, a significant expansion in terms of the frequency range of transceivers has been produced. This fact has created a severe and complex electromagnetic (EM) pollution environment. Therefore, the immunity to Electromagnetic Interferences (EMI) has become a more important constraint for integrated circuit designers. In fact, current EMC roadmaps include frequency increasing as one of the major constraints in the evolution of the immunity of integrated circuits (ICs), due to technology trend toward higher operation bandwidths provoked by customer pressure [1]. Nowadays, the most mature IC susceptibility measurement method concerning radio frequency interference (RFI) corresponds to the IEC 62132-4 Direct RF Power Injection [2]. This standard method is defined in the frequency range $150 \mathrm{kHz}-1 \mathrm{GHz}$. However, modern communication systems operation frequencies exceed the 1 $\mathrm{GHz}$ limit. Recently, several works have been focused on the frequency range expansion for DPI testing above $1 \mathrm{GHz}$ [3-4]. On the other hand, many works have been devoted to the study of the susceptibility of analog ICs (the most sensitive circuits to RFI) and, particularly, the Operational Amplifiers (OpAmps) [5-8], since they are found in a wide range of circuits where they are used to amplify and condition signals. Although, most of the proposed setups consider EMI frequencies above $1 \mathrm{GHz}$, the distributed effects of transmission lines are not deeply addressed. In this paper an alternative susceptibility characterization and modelling method for ICs is proposed in order to take into account high frequency effects and minimize their impact, such as impedance mismatches, ohmic and dielectric losses etc.

The paper is organized as follows: in Section II the proposed PCB test and measurement setup are presented. In Section III

This work was supported by the Spanish Government-MINECO under Project TEC2010-18550 and AGAUR 2009 SGR 1425. the transmission line model of the PCB as well as electrical simulation schematics and electromagnetic cosimulation layouts are provided. In Section IV the main experimental results are discussed and compared with both electrical model and electromagnetic simulations. Finally, in Section V some conclusions are summarized.

\section{TEST PCB AND EXPERIMENTAL SETUP}

\section{A. Test printed circuit board and layout criteria}

Fig. 1 shows the experimental PCB as well as the designed layout. The board (with overall dimensions $8 \times 8 \mathrm{~cm}^{2}$ ) is performed by means of a symmetrical layout based on 8-pin microstrip traces with characteristic impedance. $Z_{0}=50 \Omega$, and the same electrical length in order to present identical behavior for all injection ports. In order to investigate the RFI immunity level of several commercial OpAmps, a follower topology has been selected, as a worst EMI case [5]. Moreover, all the devices under test (DUT) are mounted on a DIP-8 package in combination with an 8 pin dual in line IC socket (808-AG11DESL-LF). The aim of this configuration is to have a worst case in terms of mismatching impedance and parasitic effects at high frequencies in order to test the injection effectiveness of the PCB. The prototype has been fabricated on the Rogers $R O 3010$ substrate (dielectric constant $\varepsilon_{r}=10.2$, thickness $h=1.27 \mathrm{~mm}, \tan \delta=0.0023$ ), with high performance up to 10 $\mathrm{GHz}$.

\section{B. Experimental setup}

Fig.2a illustrates the experimental setup. It consists of a RF signal generator (providing the RFI disturbance up to $3.2 \mathrm{GHz}$ ) directly connected to a directional coupler in order to measure

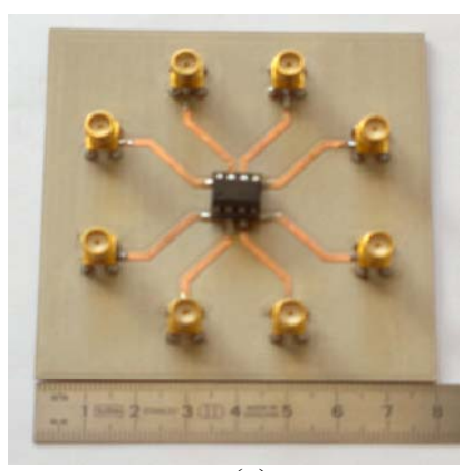

(a)

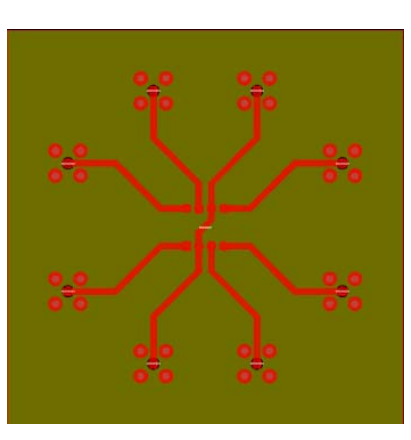

(b)
Fig. 1. (a) PCB test. (b) Designed layout. 




(a)



(b)

Fig. 2. (a) Experimental setup. (b) RFI input signal path.

the actual level of power injected into the DUT. Since the RF signal injection is applied at the noninverting input of the OpAmp (with no significant DC component) and no RF amplifier is used, it is optional to use a bias tee as a DC block. In case of RFI injection at a bias pin, the bias tee becomes mandatory in order to prevent DC supply from getting RF power. Fig. $2 \mathrm{~b}$ details the RF input signal path by taking into account all the impedance transmission line stages. The injection RFI signal will inevitably experience multiple impedance mismatches and reflections along this path, unless a good matching is performed in all intermediate stages. The objective of the test PCB is to preserve an excellent $50 \Omega$ injection path in all the system. In this case, the reflected power corresponds only to the mismatch due to the difference between the characteristic impedance of the traces, $Z_{\theta}$, and the input impedance of the $\operatorname{pin} j$ of the IC, $Z_{D U T j}$. Moreover, the rest of second order effects such as the frequency response of the directional coupler, ohmic losses, etc., have been de-embedded in order to obtain the actual value of injected power into $Z_{D U T j}$.

\section{Experimental methodology}

From the experimental return losses at the injection $j$-th SMA connector, $S_{j j}$, measured by means of a vector network analyzer, it is possible to calculate the experimental input impedance $Z_{i n j}$. In fact, for a one-port network, $S_{j j}$, is given by [9]:

$$
\mathrm{S}_{j j}=\frac{Z_{i n_{j}}-Z_{0}}{Z_{i n_{j}}+Z_{0}} .
$$

In that case, by solving (1) for $Z_{i n j}$, we obtain:

$$
Z_{i n_{j}}=Z_{0} \frac{1+S_{j j}}{1-S_{j j}} .
$$

By considering the RFI injection path as a lossless transmission line at $Z_{0}$ (a good approximation in case of all the intermediate stages, depicted in Fig. $2 \mathrm{~b}$, matched at $Z_{0}$ ), the equivalent transmission line impedance equation is given as follows:

$$
Z_{i n_{j}}=Z_{0} \frac{Z_{D U T_{j}}+j Z_{0} \tan \beta l}{Z_{0}+j Z_{D U T_{j}} \tan \beta l},
$$

where $\beta$, corresponds to the phase constant and $l$ is the length of the transmission lines $(l=29 \mathrm{~mm}$ for the designed PCB). From measured $S_{j j}$ and (3), we can determine $Z_{D U T j}$ according to:

$$
Z_{D U T_{j}}=Z_{0} \frac{Z_{0} \tan \beta l-Z_{i n_{j}}}{Z_{i n_{j}} \tan \beta l-Z_{0}}
$$

where:

$$
\beta=\frac{2 \pi}{\lambda} ; \lambda=\frac{c}{f \sqrt{\varepsilon_{r}}},
$$

being $\lambda$ and $f$ the wavelength and frequency of the propagated RFI signal and $c$ the vacuum velocity of light.

Fig. 3 depicts the methodology followed in both the measurements and simulations in order to determine $Z_{D U T i}$, and therefore, the actual degree of mismatching. The procedure is based on the application of the RFI injection port (identified as port 1) at the SMA connector corresponding to the PCB trace loaded with the noninverting input of the OpAmp $\left(Z_{D U T}\right)$. Then, by measuring $S_{11}$ and by applying equations (2-5), experimental $Z_{D U T}$ is obtained. The immunity setup is used to measure the offset voltage level at the output of the OpAmp in order to evaluate the susceptibility impact of the RFI in terms of power level and frequency. In this sense, the effectiveness of the matching in all the intermediate stages of Fig. $2 b$ is critical in order to avoid any reflection (toward the source) in the PCB except in the case of the input IC stage (intrinsic mismatching). If so, the lack of an RF amplifier in the experimental setup would be justified.

\section{MODELLING AND SIMULATION}

\section{A. Simulation methodology}

The simulation methodology is based in the obtained results from electromagnetic simulation of the PCB with the aim to perform an accurate electrical model of the transmission lines involved in the test PCB. First of all, the overall PCB layout without the IC (Fig. 1b) is electromagnetically simulated by means of the Agilent Momentum commercial software based on the well-known method of moments. An S-parameter simulation is performed by considering all ports (including SMA connectors and inputs of the IC footprint) terminated at 


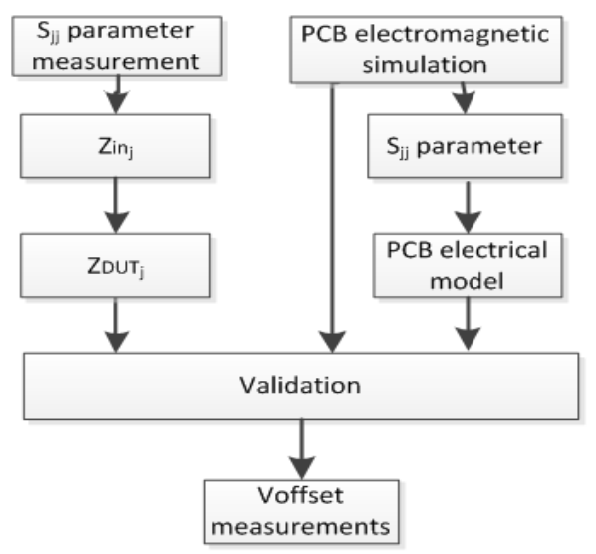

Fig. 3. Experimental and simulation methodology.

$50 \Omega$. This simulation provides the return losses $\left(\mathrm{S}_{11}\right)$ and insertion losses $\left(S_{21}\right)$ in order to determine the degree of matching of the RFI injection path. The actual width dimension of the microstrip traces $(w=1.2 \mathrm{~mm})$ is extracted by means of a commercial transmission line calculator (Agilent LineCalc) by taking into account the specifications of the substrate, detailed in Section II.

From the electromagnetic simulations it is possible to achieve the required parameters in order to develop the lumpedelement equivalent-circuit model of the PCB traces. In fact, assuming lossless transmission lines, the main design equations are [9]:

$$
v_{p}=\frac{1}{\sqrt{L C}} \quad ; \quad Z_{0}=\sqrt{\frac{L}{C}}
$$

Where $L$ and $C$ are the per-section inductance and capacitance of the transmission line cells and $v_{p}$ is the phase velocity, which can be computed from the simulated phase of $S_{21}$, $\left(S_{21}\right)$, according to:

$$
v_{p}=\frac{2 \pi l f}{\phi\left(S_{21}\right)}
$$

Once the PCB is modeled, the IC is included in the overall simulation system by including the experimental extracted Zparameters of $Z_{D U T j}$ (according to procedure detailed in Section II-C) as loads in the different transmission lines. In fact, two kinds of simulations have been performed. On the one hand, the electrical simulations of the lumped model in combination with $Z_{D U T j}$ and in the other hand, the electromagnetic cosimulation of the PCB loaded with $Z_{D U T j}$.

\section{B. Simulation setup and modelling}

Fig. 4 depicts the equivalent T-circuit model for the test PCB including the IC used in the electrical simulations, where $L_{j k}, C_{i k}$ and $Z_{D U T j}$ model the inductance, capacitance and IC's impedance of the $j$-th $\mathrm{PCB}$ trace, respectively. Table I reports the extracted per-section inductances and capacitances on the board. Notice that traces 1 and 8,2 and 7 and so on, present the same values due to the symmetry of the PCB. Anyway, the calculated values according to electromagnetic simulations are very similar because of the overall symmetry in all traces.

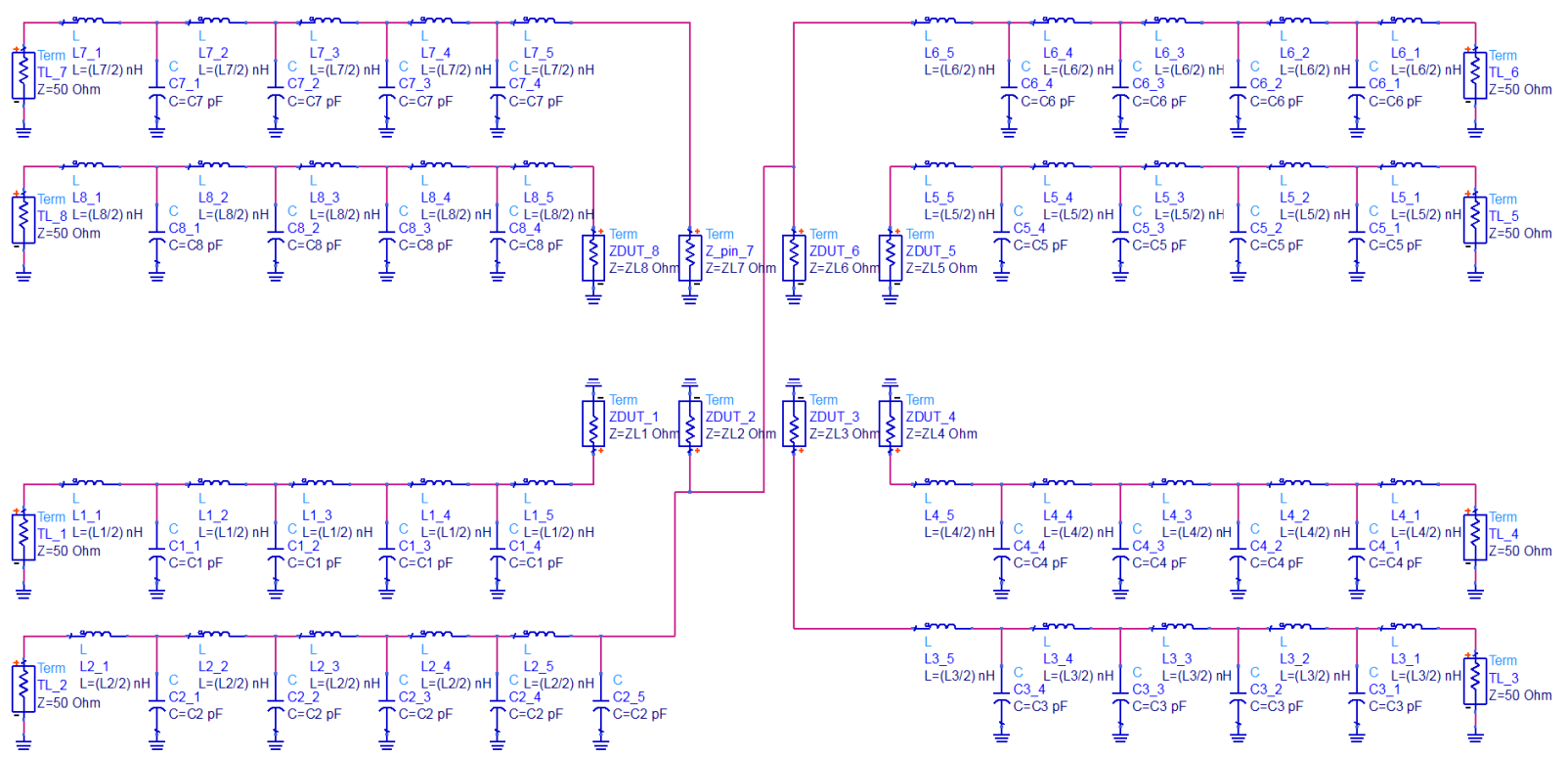

Fig. 4. Lumped equivalent circuit model for the PCB and IC used in electrical simulation. 
TABLE I. LUMPED EQUIVALENT CIRCUIT ELEMENTS OF THE PCB

\begin{tabular}{|c|c|c|}
\hline PCB Trace & $\boldsymbol{L}_{\boldsymbol{j}}(\mathbf{n H})$ & $\boldsymbol{C}_{\boldsymbol{j}}(\mathbf{p F})$ \\
\hline 1,8 & 3.265 & 1.306 \\
\hline 2,7 & 3.204 & 1.282 \\
\hline 3,6 & 3.242 & 1.297 \\
\hline 4,5 & 3.433 & 1.245 \\
\hline
\end{tabular}

Fig. 5 illustrates the cosimulation setup for the noninverting input of the OpAmp. In this case, the PCB is electromagnetically simulated in combination with the electrical model of the injection port and the experimental Zparameters obtained experimentally for $Z_{D U T j}$. In the next Section, both electrical and cosimulation results are compared with measurement data.

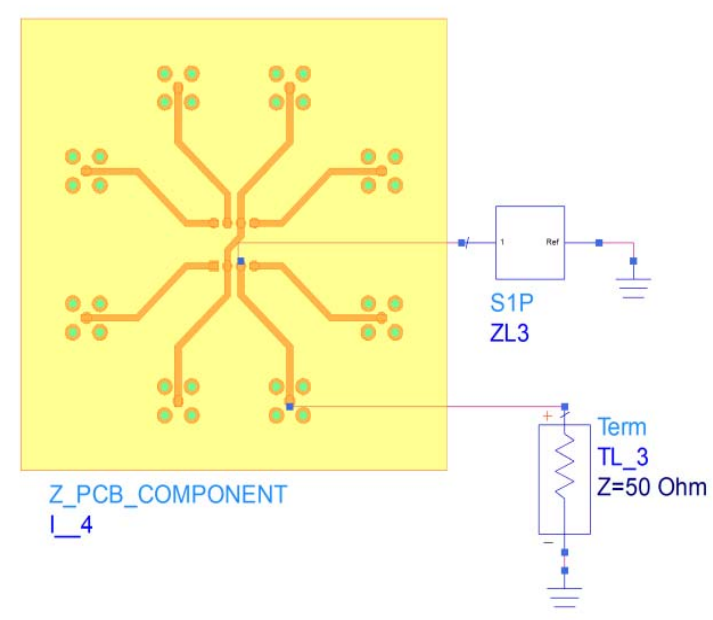

Fig. 5. Cosimulation setup $(\mathrm{PCB}+\mathrm{IC})$.

\section{RESUlts AND Discussion}

Figs. 6 and 7 include the comparison of insertion and return losses between the electrical and the electromagnetic simulations corresponding to the noninverting input injection path of the PCB terminated with $50 \Omega$. As can be observed, a good degree of matching is achieved in the PCB design for the infection frequency range $(10 \mathrm{MHz}-3 \mathrm{GHz})$ with return losses lower than $-20 \mathrm{~dB}$. Therefore, the main source of mismatch will be only due the IC, as it is desired. In order to test the setup and the degree of immunity, two commercial OpAmps with different technologies have been measured, a LM741 based on bipolar transistors and TL081 based on JFET technology. After including the OpAmps, the experimental $S_{11}$ is determined as shown in Fig. 8. It is observed the usual behavior at low frequencies where a high reflection level is produced due to the high impedance of the OpAmp. At $f>100$ $\mathrm{MHz}$, distributed effects arise and $S_{11}$ presents the corresponding behavior depending on the frequency. Obviously, a degradation of the matching is measured due to $Z_{\text {DUTj }}$. Nevertheless, $S_{11}$ levels lower than $10 \mathrm{~dB}$ can be achieved in some frequencies ranges above $1 \mathrm{GHz}$.



Fig. 6. Simulated insertion losses concerning the injection path of the noninverting input of the OpAmp, terminated at $50 \Omega$ (with no IC).

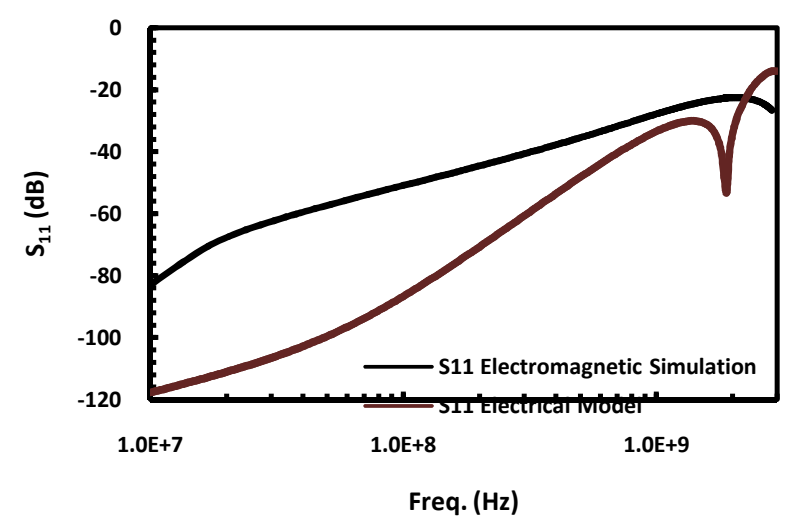

Fig. 7. Simulated return losses concerning the injection path of the noninverting input of the OpAmp, terminated at $50 \Omega$ (with no IC).

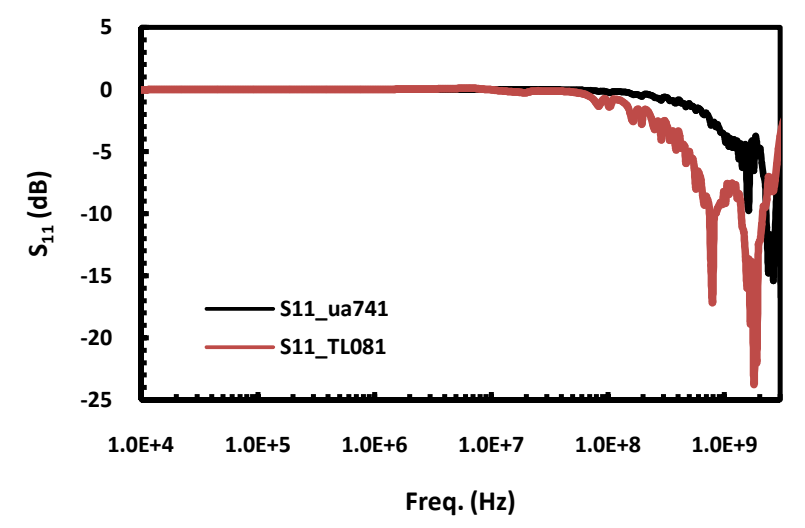

Fig. 8. Experimental return losses for the noninverting input of the PCB loaded with the tested OpAmps.

By using equations (2-4) we can determine experimental input impedance of the DUT as shown in Fig. 9. Figs. 10 and 11 show the comparison between the measured input impedance $Z_{\text {in }}$ and simulated results by means of the electrical model and the electromagnetic cosimulation setup explained in the 


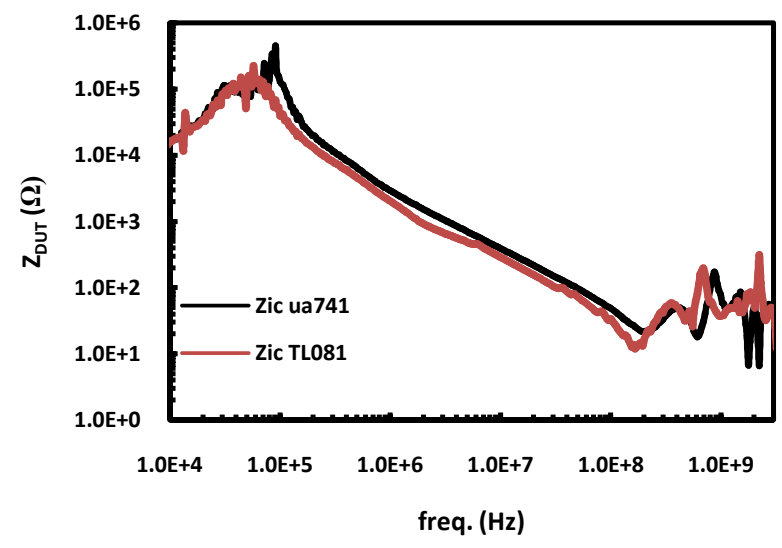

Fig. 9. Experimental $Z_{D U T}$ of both tested OpAmps.

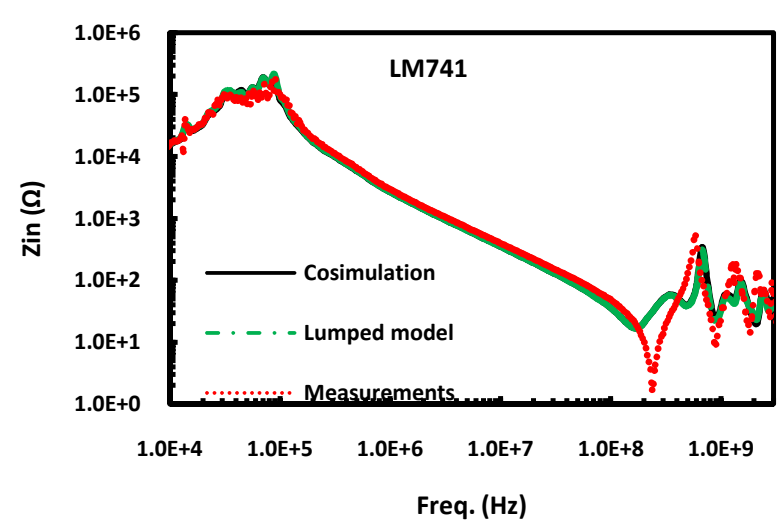

Fig. 10. Input impedance measured at the injection port SMA of the PCB in comparison with lumped model simulation and electromagnetic cosimulation for the LM741 OpAmp.

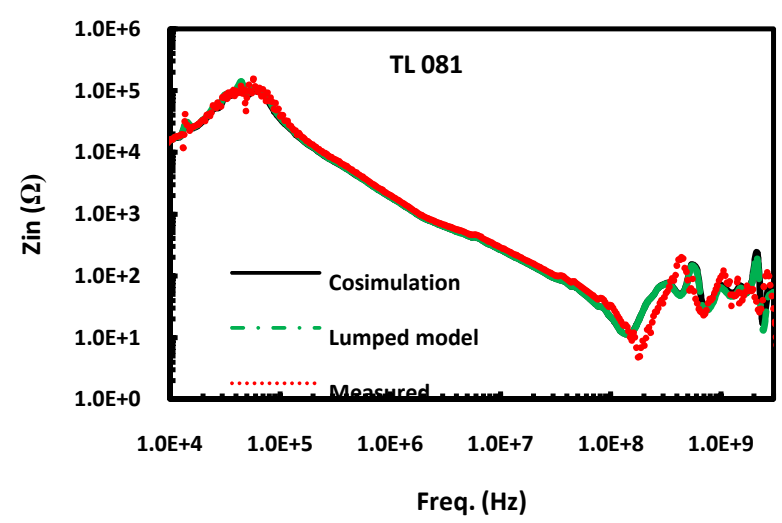

Fig. 11 . Input impedance measured at the injection port SMA of the PCB in comparison with lumped model simulation and electromagnetic cosimulation for the TL081 OpAmp.

previous Section. A good degree of accuracy is achieved between both simulations and experimental data. Since the lumped model is obtained from the electromagnetic



Fig. 12. Effect of RFI injected at the input SMA of the PCB loaded with the LM741 OpAmp at room temperature.

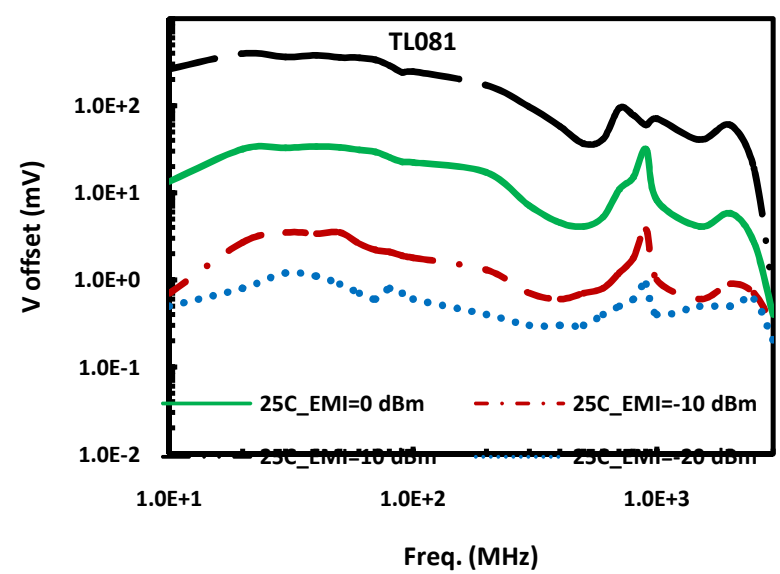

Fig. 13. Effect of RFI injected at the input SMA of the PCB loaded with the TL081 OpAmp at room temperature.

simulations, an excellent accuracy between both simulation results is achieved. Therefore, the level of accuracy between simulations and experimental is achieved thanks to the good performance of the electromagnetic simulations. These results validate the modeling and simulation process with regard to the proposed experimental setup. In order to test the proposed experimental setup, the offset voltage level of both OpAmps has been measured at the output pin of the corresponding ICs. Figs. 12 and 13 depict the frequency impact of the offset by injecting several levels of RFI power at room temperature. It is observed that high levels of voltage offset are reached. For instance, for the LM741 an offset level higher than $100 \mathrm{mV}$ is achieved for a $0 \mathrm{dBm}$ RFI power. According to measured conventional offset level (measured value is $1.2 \mathrm{mV}$ ), a susceptibility impact of the EMI case higher than 2 order of 
magnitude is observed. In the case of the TL081 OpAmp, similar results are obtained. In fact, the free EMI case offset corresponds to $0.8 \mathrm{mV}$ whereas a maximum value for a 10 $\mathrm{dBm}$ RFI power imply an offset in the order of $100 \mathrm{mV}$. In all cases, the losses and mismatching have been de-embedded in the experimental setup. Therefore, the proposed setup prove experimentally that RF amplifier is not required if a good matching is achieved on RFI injection port for the tested ICs.

\section{CONCLUSION}

In this work a matched an symmetrical test PCB has been presented in order to characterize the RFI susceptibility at frequencies above $1 \mathrm{GHz}$. The system consists of minimizing the mismatching in all the stages of the injection path in order to simplify the overall experimental setup by not considering a RF Amplifier. Both electrical simulations and electromagnetic cosimulations validate the experimental results concerning measured impedances. Moreover, the method has been checked for the susceptibility impact into 2 commercial OpAmps by implementing a follower topology.

\section{REFERENCES}

[1] M. Ramdani, E. Sicard, A. Boyer, S. Ben Dhia, J.J. Whalen, T.H. Hubing, M. Coenen and O. Wada, "The electromagnetic compatibility of integrated circuits-past, present and future", IEEE Transactions on Electromagnetic Compatibility, Vol. 51, pp. 78-100, February 2009.

[2] EN IEC 62134-4, Integrated Circuits - Measurement of electromagnetic immunity, $150 \mathrm{kHz}$ to $1 \mathrm{GHz}$ - Part4: Direct RF Power injection method.

[3] J. Catrysse, D. Pissoort and F. Vanhee, "Expanding the frequency range for DPI testing of IC's above $1 \mathrm{GHz}$ : an alternative proposal", Proc. of the EMC Europe 2011, pp. 400-404, September 2011, York, UK.

[4] S. Buntz, M. Blatter, H. Leier, S. Fuchs, D. Gwissdalla and W. Menzel, "Direct power injection immunity measurements at frequencies above 1 GHz", Proc. of the EMC Europe 2006, vol. 2, pp. 882-887, September 2006, Barcelona, Spain.

[5] A. Richelli, "Measurements of EMI susceptibility in ultra-low-voltage OpAmps", Proc. of the EMC Compo 2011, pp. 13-17, November 2011, Dubrovnik, Croatia.

[6] F. Fiori, "Design of an Operational Amplifier Input Stage Immune to EMI," IEEE Transactions on Electromagnetic Compatibility, vol.49, no.4, pp.834-839, November 2007.

[7] J. Gago, J. Balcells, D. Gonzalez, L. Lamich, J. Mon, A. Santolaria, "EMI Susceptibility Model of Signal Conditioning Circuits Based on Operational Amplifiers," IEEE Transactions on Electromagnetic Compatibility, vol.49, no.4, pp.849-859, November 2007

[8] J.-M. Redoute, M. Steyaert, "Measurement of EMI induced input offset voltage of an operational amplifier," Electronics Letters , Vol.43, no.20, pp.1088-1090, September 2007.

[9] D.M. Pozar, "Microwave Engineering, $4^{\text {th }}$ Edition", John Wiley \& Sons, 2012. 